Klaus Schmidt

\title{
Die megalithischen Kreisanlagen des steinzeitlichen Göbekli Tepe
}

This article deals with the round structures built on Göbekli Tepe (present-day southeastern Turkey) during the transition from the Epipalaeolithic to the Neolithic period. An important result of the excavations carried out in recent years has been the discovery of a large number of T-shaped, monolithic pillars, which, due to the occasional presence of highly stylized arms and hands depicted in bas-relief, have been positively identified as anthropomorphic stone beings. These pillar-like beings, some over five meters tall, encircle and define this space. Within the space, ritual acts were performed, acts in which liquids have been shown to have played a major role. At the moment, it seems that no precise definition can be given of the relation between the pillar-like beings (including the animals occasionally depicted on them) and the acts that were performed here. But the structures on Göbekli Tepe - like those of many other cultures - may well have been erected in the context of burial rites and in the service of a culture that seeks to triumph over death.

Weltweit lebten die Menschen der Altsteinzeit als Wildbeuter, denn die nahrungsproduzierende Lebensweise und die hierfür notwendigen Grundlagen, darunter am wichtigsten: Kulturpflanzen und Haustiere, waren ihnen noch unbekannt. Es war >die große Zeit der Eiszeitjäger<, die mit viel Know-how den Herausforderungen der im Pleistozän oft lebensfeindlichen Umwelt trotzten. Mit der >Eiszeitkunst< hinterließen sie uns beeindruckende Zeugnisse einer vielfältigen spirituellen Welt. Naturheilige Plätze, spezielle landschaftliche Formationen, Felsschutzdächer, Höhlen oder auch nur besondere Bäume markierten die Orte, die für die Durchführung sakraler Handlungen genutzt wurden.

Auch in der Nacheiszeit, im Holozän, verharren die Menschen in den meisten Teilen der Welt zunächst auf der wildbeuterischen Stufe. In der >Alten Welt< wird diese Phase als Mittlere Steinzeit, als Mesolithikum, bezeichnet. Es folgt die Jüngere Steinzeit, das Neolithikum, dessen wichtigste Neuerung die Nahrungsproduktion darstellt, also eine Änderung der Subsistenzstrategien mit dem Wechsel vom nomadisierenden Wildbeutertum hin zum sesshaften und bäuerlichen Leben. Das Auftreten der neolithischen Lebensweisen erfolgt allerdings in verschiedenen Teilen der Erde zu verschiedenen Zeiten und teilweise unabhängig voneinander. Für den Fortgang der Geschichte in der >Alten Welt< ist der fruchtbare Halbmond Vorderasiens ausschlaggebend. Hier findet in der Zeit um 9000 v. Chr. weltweit gesehen das erste Mal dieser Übergang statt, der die elementaren Grundvoraussetzungen für die späteren urbanen Zivilisationen der Antike bereitet. Die Kernzone der Neolithisierung liegt hierbei im Herzen des fruchtbaren Halbmonds zwischen den Oberläufen von Euphrat und Tigris. Es handelt sich um eine Landschaft, in der die Verbreitungsgebiete der Wildformen all der Pflanzen und Tiere, die im 9. Jt. v. Chr. domestiziert wurden, eine gemeinsame Schnittmenge finden, denn die Domestikation einer Spezies ist natürlich nur in der Heimat der jeweiligen Wildform denkbar. Hier in Obermesopotamien begannen am Ende des 10. Jts. v. Chr. die Menschen mit der Domestikation von Pflanzen und Tieren das sog. neolithische Paket zu schnüren, dessen Besitz 
der neuen bäuerlichen, nahrungsproduzierenden Lebensweise zum schnellen Siegeszug und zur Ausbreitung über ganz Europa und Mittelasien hinweg verhelfen sollte.

In dieser Kernzone der Entstehung nahrungsproduzierender Lebensformen liegt ein Platz, der unmittelbar nach dem Ende der Eiszeit in der 1. Hälfte des 10. Jts. v. Chr. mit einer spektakulären Befundlage auf den Plan tritt. Es handelt sich um den seit 1995 vom Deutschen Archäologischen Institut erforschten Göbekli Tepe'. Hier beschränkt sich die Schöpferkraft des Menschen nicht mehr auf den Auftritt auf einer von der Natur bereiteten Bühne. Erstmals in seiner Geschichte setzt sich der Mensch als Baumeister monumentaler Anlagen in Szene und das Verlangen nach einem >sakralen Raum< wird zu einem gewaltigen Gebilde aus unterschiedlichsten und oft monumentalen Bauteilen aufgetürmt.

Errichtet wurden mehrere in ihrer Konzeption weitgehend gleiche Anlagen, die von einem immer wiederkehrenden Element beherrscht werden: den T-förmigen, aus Kalkstein gefertigten, monolithischen Pfeilern, die kreis- oder ovalförmig um zwei besonders große Pfeiler im Zentrum der Anlage aufgestellt wurden (Abb. 1). Der Begriff »Pfeiler« wird hierbei im Sinn der heiligen Pfeiler Altägyptens benutzt, deren bekannteste Vertreter die Obelisken bilden. Diese Pfeiler mussten bekanntermaßen niemals die Last eines darüber aufgetürmten Gebälks und eines Daches tragen, sie ragten völlig unbeschwert und frei in den Himmel. Ob dies auch für die T-Pfeiler des Göbekli Tepe zutrifft, ist allerdings weiterhin noch nicht sicher: Die Frage, ob wir es mit offenen, hypäthralen oder mit überdachten, die T-Pfeiler möglicherweise als Stützen benutzenden Anlagen zu tun haben, ist noch nicht abschließend geklärt. Sie kann aber aus Platzgründen in dem vorliegenden Aufsatz auch nicht weiter vertieft werden. Bei der Behandlung des Themas »Bild Raum - Handlung« soll im Fall des Göbekli Tepe und der sakralen Anlagen des frühholozänen Obermesopotamiens insgesamt die Frage der Überdachung - ob überhaupt und wenn ja, in welcher Gestalt - ausgeklammert sein.

Die Steinkreise des Göbekli Tepe ragen nicht nur durch ihre Monumentalität, nicht nur durch die Vielfalt ihrer künstlerischen Ausstattung mit großformatigen Skulpturen und Reliefs aus den bisher bekannten sheiligen Stätten der Steinzeit< hervor, sie scheinen vielmehr eine Schüsselfunktion im Transformationsgeschehen vom Wildbeuter zum Bauern zu besitzen. Denn die Steinkreise bezeugen mehr als nur die Fähigkeit zur Mobilisierung großer Menschengruppen, ohne deren Arbeitskraft die tonnenschweren Pfeiler nicht hätten hergestellt, transportiert und aufgerichtet werden können.

Oft finden sich auf den T-Pfeilern des Göbekli Tepe in Flachrelief dargestellt Tiere, die einzeln oder in verschiedener Kombination vorkommen können, so z. B. Schlangen und Skorpione, Füchse und Wildschweine, Gazellen und Wildesel. Daneben gibt es aber auch abstrakte Symbole. Diese können bisher nicht gedeutet werden, sie illustrieren aber 


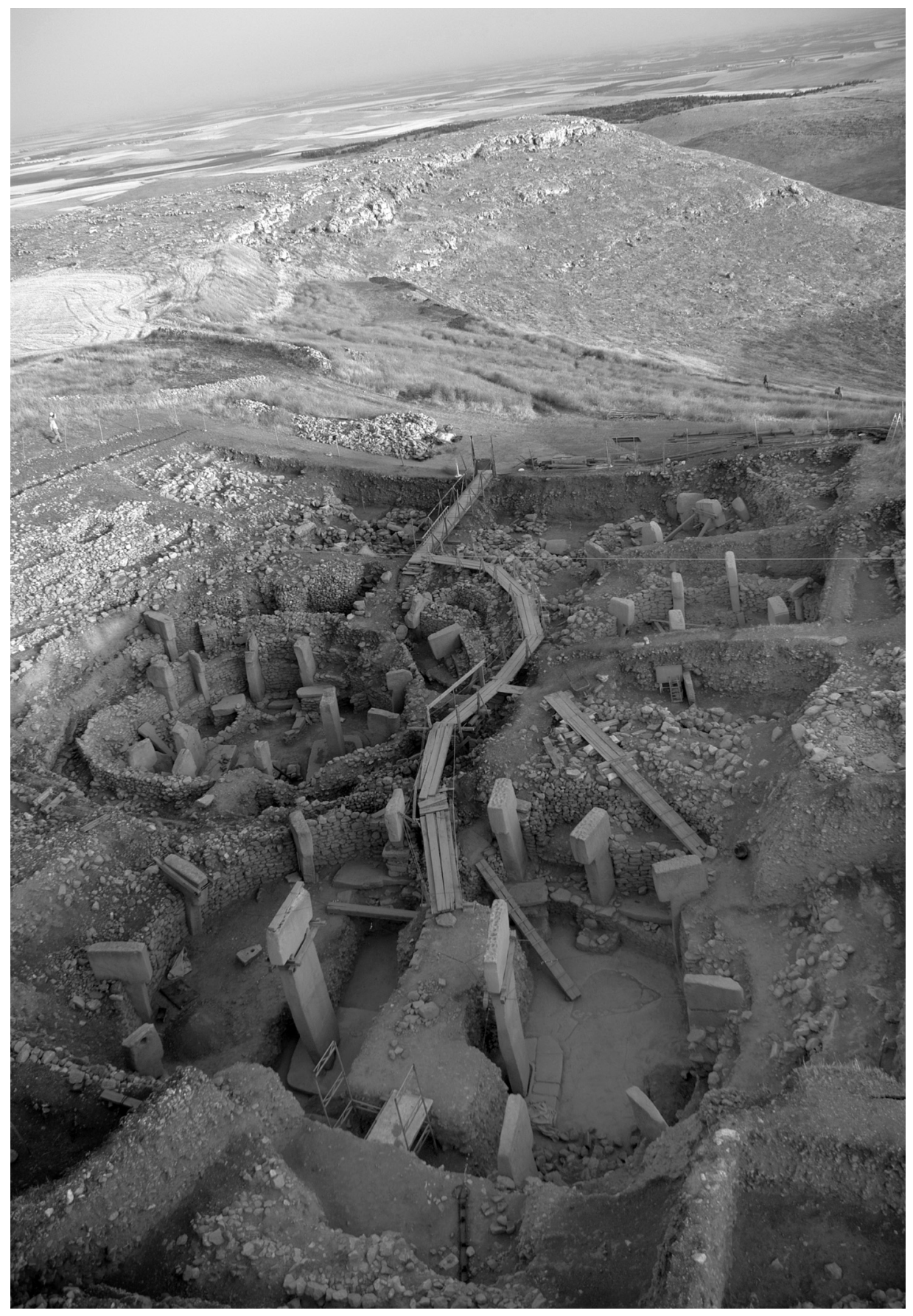

Abb. 1 | Göbekli Tepe, das Hauptgrabungsgelände am Südhang mit den monumentalen Anlagen A bis D im Frühjahr 2010 von Norden aus gesehen. 
zusammen mit den Tieren offenbar mythische Geschehen, die auf den Pfeilern abgebildet sind und mit diesen Tieren wahrscheinlich in Verbindung stehen.

Der Inhalt dieser steinzeitlichen Mythen wird für uns nie mehr als in vagen Umrissen zu erschließen sein und wir können nur vermuten, dass auch hier wie in den durch mündliche oder schriftliche Überlieferung bekannten Mythen der Welt wichtige, letztlich kosmogonische Fragen im Mittelpunkt des Geschehens stehen, wobei wir aber durchaus auch manch pikareske Erzählmotive erwarten können. Auch wenn uns beim Versuch, anhand der überlieferten Bilder die steinzeitlichen Mythen gleichsam wiederzubeleben, enge und nicht ablegbare Fesseln angelegt sind, so können wir doch die Bedeutung der T-Form der Pfeiler glücklicherweise mit großer Sicherheit festmachen. Aufgrund der am Pfeilerschaft manchmal in Relief ausgeführten Arme und Hände sind die Pfeiler als zwar stark stilisierte, dennoch menschengestaltige Figuren zu verstehen. Der T-Kopf bildet den Kopf in Profilansicht, der Pfeilerschaft den Körper. Zwei auf der Bauchseite befindliche, in Flachrelief angegebene Bänder, können als Bekleidungsbestandteil in der Art einer Stola verstanden werden. Da sich die Stola wesentlich öfter als Arme und Hände beobachten lässt, kann man auch für die Pfeiler, bei denen die Darstellung von Armen und Händen fehlt, von einer Bauch- bzw einer Rückenseite sprechen. Deshalb ist festzustellen, dass alle Pfeiler des Kreises auf die Zentralpfeiler hin ausgerichtet sind.

Es gilt demzufolge festzuhalten, dass es sich bei den Pfeilern also weniger um architektonische Bauelemente handelt als um Statuen - zwar hochstilisierte Statuen in einer geradezu paläokubistisch zu nennenden Stilrichtung, die erst im frühen 20. Jahrhundert unserer Zeit wieder aufgegriffen wird (ohne dabei zur T-förmigen Stilisierung des menschlichen Körpers zu finden) - und dass es sich bei den Pfeileranlagen weniger um Bauwerke handelt als um Steinkreise. Im Unterschied zu den großen Steinkreisen des atlantischen Europas verbinden allerdings Mauern die im Kreis stehenden Pfeiler, so dass das Innen und Außen deutlich voneinander geschieden werden. Völlig frei stehend aufgestellt sind nur die beiden Zentralpfeiler, die den Raum dominieren. Dass es hier um einen sakralen Raum geht, kann dabei kaum bezweifelt werden.

Bemerkenswert ist die Gesichtslosigkeit der Pfeilerwesen. Nie sind Augen oder andere Züge des menschlichen Gesichts dargestellt, immer verbleibt die >Gesichtsfläche< ohne anatomische Details. Allerdings ist sie nicht immer völlig leer. Bei mehreren Pfeilern finden sich Tierreliefs auf der Vorderseite der T-Köpfe, in einem Fall auch ein abstraktes H-förmiges Symbol. In jeden Fall sollte das durchgängige Fehlen anatomischer, das Gesicht wiedergebender Details auf den T-Köpfen als ein Merkmal verstanden werden, das zusammen mit der Monumentalität der teilweise über $5 \mathrm{~m}$ großen steinernen Wesen eines anzeigt: Sie sind riesig und entrückt, sie verkörpern Mächte, die ganz offensichtlich einer anderen Welt entstammen, sie gehören einer höheren Sphäre als der diesseitigen an. Die Frage, ob es sich um Götter handelt oder um Ahnen, um Geister oder Dämonen, kann natürlich nicht ohne Weiteres beantwortet werden und im vorliegenden Zusammenhang wird sie auch nicht weiter thematisiert. Denn wir müssen uns in der Frage, wen die T-Pfeiler 
verkörpern, nicht verbindlich entscheiden, wenn wir der Grundaussage zustimmen: Es handelt sich in jeden Fall um sehr wichtige Wesen, die sich dort im Kreis versammeln. $\mathrm{Zu}$ den beiden ersten Elementen der Thematik »Bild - Raum - Handlung « können wir daher mit - vielleicht überraschender - Klarheit wichtige Beobachtungen festhalten. Der sakrale Raum des frühholozänen Obermesopotamiens wird von einer (mehr oder weniger) kreisförmigen Versammlung hochstilisierter menschengestaltiger, ganz offenbar einer jenseitigen Welt zugehöriger Wesen gebildet, die in streng kubischer Form in Stein gehauen mit teilweise über $5 \mathrm{~m}$ Höhe die ersten Monumentalstatuen der Menschheit darstellen. Mit Mauern verbunden bilden sie den sakralen Raum und grenzen das Außen vom Innen deutlich ab. Das sakrale Geschehen findet nicht umrahmt von einem Bauwerk statt, die Versammlung selbst formiert sich zum architektonischen Gebilde. Die Bildwelt der Kreisanlage lässt sich hierbei in drei Ebenen gliedern: zuerst die der anthropomorphen T-Pfeiler selbst, die das Geschehen fraglos dominieren, dann die auf den Pfeilern angebrachten Tierreliefs, die mythologische Geschehen zu illustrieren scheinen, und als dritte Ebene die der >kleinen Zeichen<, die neben Tiermotiven auch abstrakte Symbole beinhalten ${ }^{2}$. Alle drei Ebenen kodieren einen kulturellen Text, der für den steinzeitlichen Menschen lesbar war und der den architektonischen Strukturen und dem so geschaffenen sakralen Raum in großer Intensität aufgeprägt ist.

Auch zum dritten Teil des Themas, dem Aspekt der »Handlung«, lässt sich Einiges beitragen, und dies gleich auf zwei Ebenen. Zunächst sind im Inneren der Kreise rituelle Handlungen der steinzeitlichen Menschen zu erkennen. Was da genau stattfand und welche Zwecke damit verfolgt wurden, ist im Dunkel der schriftlosen Vergangenheit natürlich allenfalls noch zu erahnen, doch fand >etwas statt, denn es gibt Installationen und Befunde, die dies deutlich anzeigen. Bei diesen Handlungen scheinen Flüssigkeiten eine wichtige Rolle zu spielen, da sich mehrfach Steingefäße vor und zwischen den Zentralpfeilern beobachten ließen. In Anlage B befindet sich unmittelbar westlich vor dem östlichen Zentralpfeiler in den Terrazzoboden eingelassen eine flache Kalkschale, in die ein kleiner, in den Rand der Schale eingesenkter Kanal führt (Abb. 2). Ganz offenbar konnte so eine Flüssigkeit in die Schale geleitet werden. Ähnliche Schalen mit Zuleitungskanal im Schalenrand sind am Göbekli Tepe aus sekundärem Kontext mehrfach belegt. Sie erhielten angesichts des Befundes in Anlage B im Grabungsjargon den Begriff »Opferschalen«. Das Fassungsvolumen der Schalen liegt dabei allerdings deutlich unter einem Liter, so dass es sich nicht um große Mengen vergossener Flüssigkeiten gehandelt haben kann.

In der ovalen Anlage $\mathrm{C}$ wurde im Fußboden keine derartige >Opferschale< angetroffen. Den Fußboden bildet hier der gewachsene Fels, der sorgfältig geglättet und offenbar um mindestens $30 \mathrm{~cm}$ abgearbeitet wurde, da im Zentrum des Ovals zwei entsprechend 
hohe, trapezförmige Podeste zur Aufstellung der Zentralpfeiler von der Absenkung ausgespart blieben. Auf der westlichen Fläche des östlichen Podests befinden sich zwei kreisförmige schalenartige Einsenkungen. Ihnen könnte die Funktion der sog. Opferschale von Anlage B zukommen. Zusätzlich fanden sich auf dem Podest zwei in der Mitte durchlochte Steinteller und ein napfförmiges Steingefäß, dazu noch die Skulptur eines deutlich unterlebensgroßen Keilers (Abb. 3). Die hier durchgeführten Rituale beinhalteten offenbar recht umfangreiche, detailreiche Geschehen.

Die Bedeutung des Raums vor und zwischen den Zentralpfeilern im Hinblick auf rituelle Geschehen setzt sich in Anlage F fort. Die Zentralpfeiler sind hier im Unterschied zu den Anlagen A bis E nicht nach Südsüdost, sondern nach Südwest hin orientiert. Vor dem südöstlichen Zentralpfeiler treffen wir auf ein zwar zerbrochenes, ursprünglich aber voluminöses Gefäß. In den Anlage A und D ist der Bereich zwischen den Zentralpfeilern noch nicht bis zum Fußboden ausgegraben - es sollte keine Überraschung sein, wenn auch hier weitere Gefäße angetroffen werden.

Die Verwendung von Flüssigkeiten im Rahmen der in den Kreisanlagen vollführten Rituale erklärt möglicherweise auch das offenkundige Bestreben, die Anlagen mit wasserfesten Fußböden auszustatten. In den Anlagen C bis E ist es der sorgsam geglättete gewachsene Fels, bei Anlage B, F und G ein betonartiger, ebenfalls wasserfester Terrazzoboden (in Anlage A wurde der Fußboden noch nicht freigelegt). Die Annahme, dass die Terrazzoböden Felsböden nachzuahmen versuchen, ist dabei sehr naheliegend. Doch ist mit diesen Beobachtungen das Spektrum der in den Anlagen vollführten Rituale bei weitem noch nicht durchmessen und es werden noch viele andere Dinge von Bedeutung gewesen sein.

Eine zweite »Handlungs«-Ebene eröffnen die T-Pfeiler selbst. Auch wenn nicht viele mit der in Flachrelief ausgeführten Darstellung von Armen und Händen versehen sind, so kann kein Zweifel daran bestehen, dass die anthropomorphe Gestalt für alle T-Pfeiler gilt und dass der Gestus der V-förmig auf den Bauch gelegten, sich gegenüberstehenden und sich nicht berührenden Hände von allen T-Pfeiler eingenommen wird.

Gestische Kommunikation gehört zu den wichtigen Themen der Kognitionsforschung. Leider ist der V-förmige Handgestus der T-Pfeiler noch wenig erforscht, ein Sachverhalt, der um so bedauerlicher ist, als ihm beim Verständnis der Kreisanlagen des Göbekli Tepe eine Schlüsselfunktion zukommt. Die Handdarstellung für sich genommen ist ein vieldeutiges Zeichen. Das Spektrum der Deutungsmöglichkeiten reicht von der Gottes- zur Beterhand, von der Abwehr- zur Grußhand und im anderen Deutungsextrem zur abgeschlagenen Hand des Feindes als Siegeszeichen 3 . Eine grundsätzlich polyseme Form erlaubt es dem fernen Betrachter nicht, sich auf eine ganz konkrete Deutung festzulegen. Die Vieldeutigkeit des Handzeichens wird allerdings in Fall der T-Pfeiler in ihrer Spannweite erheblich reduziert. Denn es erscheint die Hand ja nicht als Bildzeichen, sondern als Bild, anatomisch eingebunden in die kubische Körperlichkeit der Pfeilerwesen. Ohne

Vgl. z. B. Jursch 1952; Wollenik 1982. 


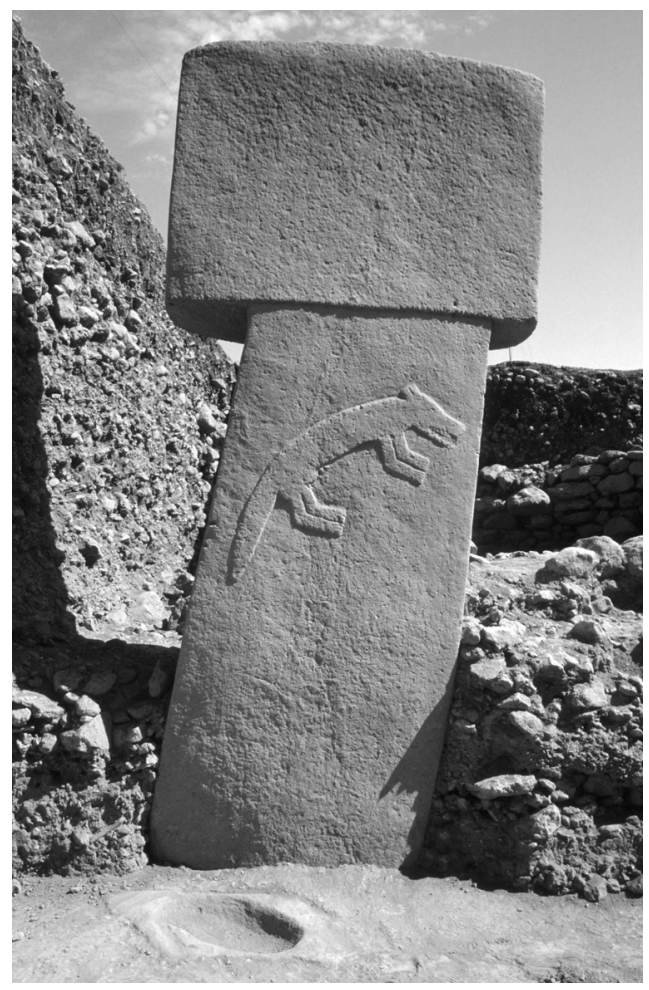

Abb. 2 | Der östliche Zentralpfeiler der Anlage B mit einer in den Terrazzoboden eingelassenen Kalkschale.

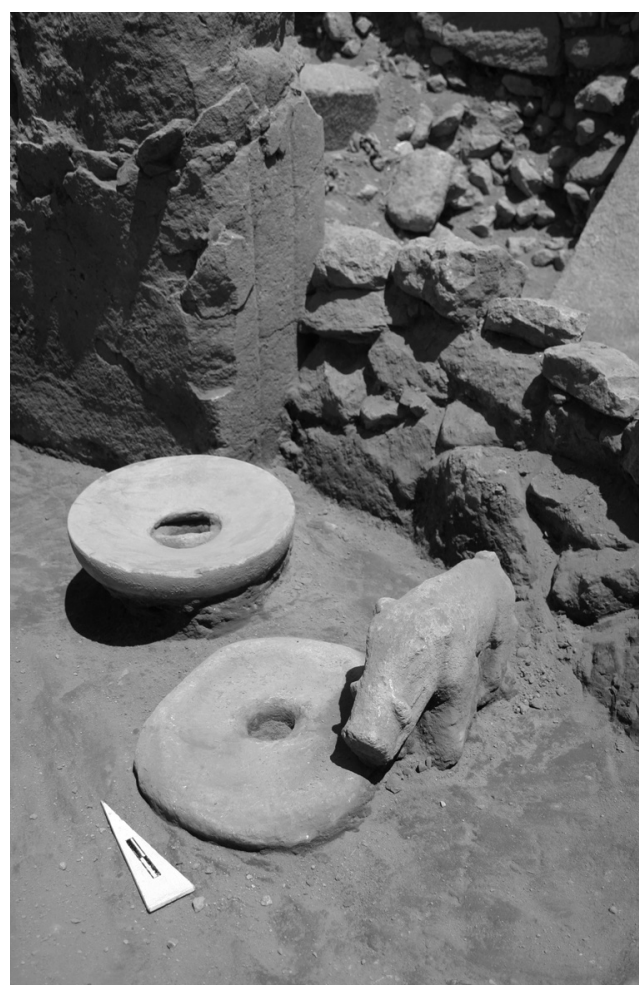

Abb. 3 | Anlage C, auf dem östlichen Felspodest zwei in der Mitte durchlochte Steinteller und ein napfförmiges Steingefäß, dazu noch die Skulptur eines deutlich unterlebensgroßen Keilers.

Frage bemerkenswert ist, dass alle Pfeiler offenbar die gleiche Geste zeigen, denn wir können, wie schon angeführt, recht sicher sein, dass auch die Pfeiler ohne Arm- und Handdarstellungen letztlich den gleichen Gestus einnahmen, einen Gestus, der so selbstverständlich war, dass auf seine bildliche Umsetzung eben auch verzichtet werden konnte.

Ist mit diesem Gestus Meditation, ist Gebetshaltung gemeint? Die Welt des Buddhismus z. B. kennt eine Vielzahl von Handgesten ${ }^{4}$, doch fehlt der Gestus der V-förmig sich gegenübergestellten Hände. Auf dem Bauch übereinander gelegte Hände sind in der Malerei des Mittelalters oft bei Frauendarstellungen zu beobachten. Dies wird als Ausdruck weiblicher Schamhaftigkeit verstanden. Aber die Hände der T-Pfeiler sind nicht ruhend übereinander gelegt, sondern stehen sich gegenüber, und es erscheint als sehr wahrscheinlich, dass dieser Unterschied nicht im darstellerischen Unvermögen der 
steinzeitlichen Künstler begründet liegt. Die auffällig positionierten Hände scheinen neben der Stola das einzige darstellungsrelevante Merkmal der T-Pfeiler zu sein (Abb. 4). Durch die Armhaltung könnte man nun annehmen, dass die Hände zur Geschlechtsregion hingeführt werden. Zeigen die Hände etwa auf den Phallus- bzw. die Vagina, auch wenn kein Geschlechtsteil abgebildet ist?

Bei der Suche nach Vergleichen zur V-förmigen Handhaltung der T-Pfeiler erinnern wir uns schnell der Monumentalstatuen der Osterinsel, die in ganz ähnlicher Weise in Flachrelief dargestellte und am Körper anliegende, nach vorn gebeugte Arme und auf den Bauch gelegte und sich gegenüberstehende, aber nicht berührende Hände besitzen ${ }^{5}$. Doch auch im Fall der Osterinselstatuen, über die wir in vielen Aspekten, so z. B. hinsichtlich ihrer Herstellung und ihres Transports von den Steinbrüchen zu den Aufstellungsorten hin, gut unterrichtet sind, ist wenig über die Bedeutung des an sich auffälligen HandGestus bekannt. So ist nicht völlig klar, ob die Hände ruhen oder ob sie etwas halten, etwas präsentieren, denn aus den vorhandenen Abbildungen wird oft nicht ersichtlich, ob es sich bei bestimmten Objekten, die sich oft zwischen den Händen beobachten lassen, nur um die Knoten eines Hüfttuches handelt oder ob auch das männliche Glied abgebildet ist - und ob sich dieses im eregierten Zustand befindet. So ist sich selbst Heide-Margaret Esen-Baur, die über die Statuen gearbeitet hat, hier offenbar nicht vollkommen sicher und und sie findet zu den Worten: »Zwischen den Händen ist der Knoten des Lendenschurzes, gelegentlich das männliche Glied in Hochrelief gearbeitet [...] Vom Gesamteindruck her dürfte es sich bei den Kollossalfiguren um phallische Bildnisse handeln ${ }^{6}$.

Wenig beachtet wurde auch der Sachverhalt, dass die >klassischen< Monumentalstatuen der Osterinsel knapp unterhalb der Hände gleichsam abgeschnitten, Unterkörper und die Beine nicht dargestellt wurden. Die Unterseite der Statuen ist völlig gerade gebildet, so dass angesichts der großen planen Fläche eine stabile Aufstellung ohne Verankerung möglich war. Auch die steinzeitlichen T-Pfeiler besitzen keine Beine. Sie enden zwar nicht unmittelbar unterhalb der Hände, doch zeigen die Proportionen von Kopf, Oberkörper, Bauch (hier ruhen die Hände) und Schaftunterteil unzweideutig, dass auch hier der untere Körperabschnitt mit den Beinen allenfalls rudimentär und stark verkürzt dargestellt wurde. Der Erkenntniswert der aufgezeigten phänomenologischen Gemeinsamkeiten der Monumentalplastik beider geographisch und zeitlich entfernter Regionen muss vorläufig leider unbestimmt bleiben. Das Beispiel »Osterinsel« wurde deshalb hier ausgebreitet, um aufzuzeigen, dass selbst im Fall der durch ethnographische Daten gut erschlossenen Osterinsel und ihrer Monumentalstatuen der Hand-Gestus der sich gegenübergestellten, sich aber nicht berührenden Hände noch nicht abschließend erforscht zu sein scheint.

So verwundert es nicht weiter, dass wir auch bei den steinzeitlichen Vergleichsstücken, die diesen Hand-Gestus aufweisen, über dessen Bedeutung vorläufig keine völlige

6 Esen-Baur 1989, 92 und 93. 
Abb. 4 | Der östliche Zentralpfeiler der Anlage D mit Armen, Händen, Gürtel und Lendenschurz, Höhe mit Felspodest 5,5 m.

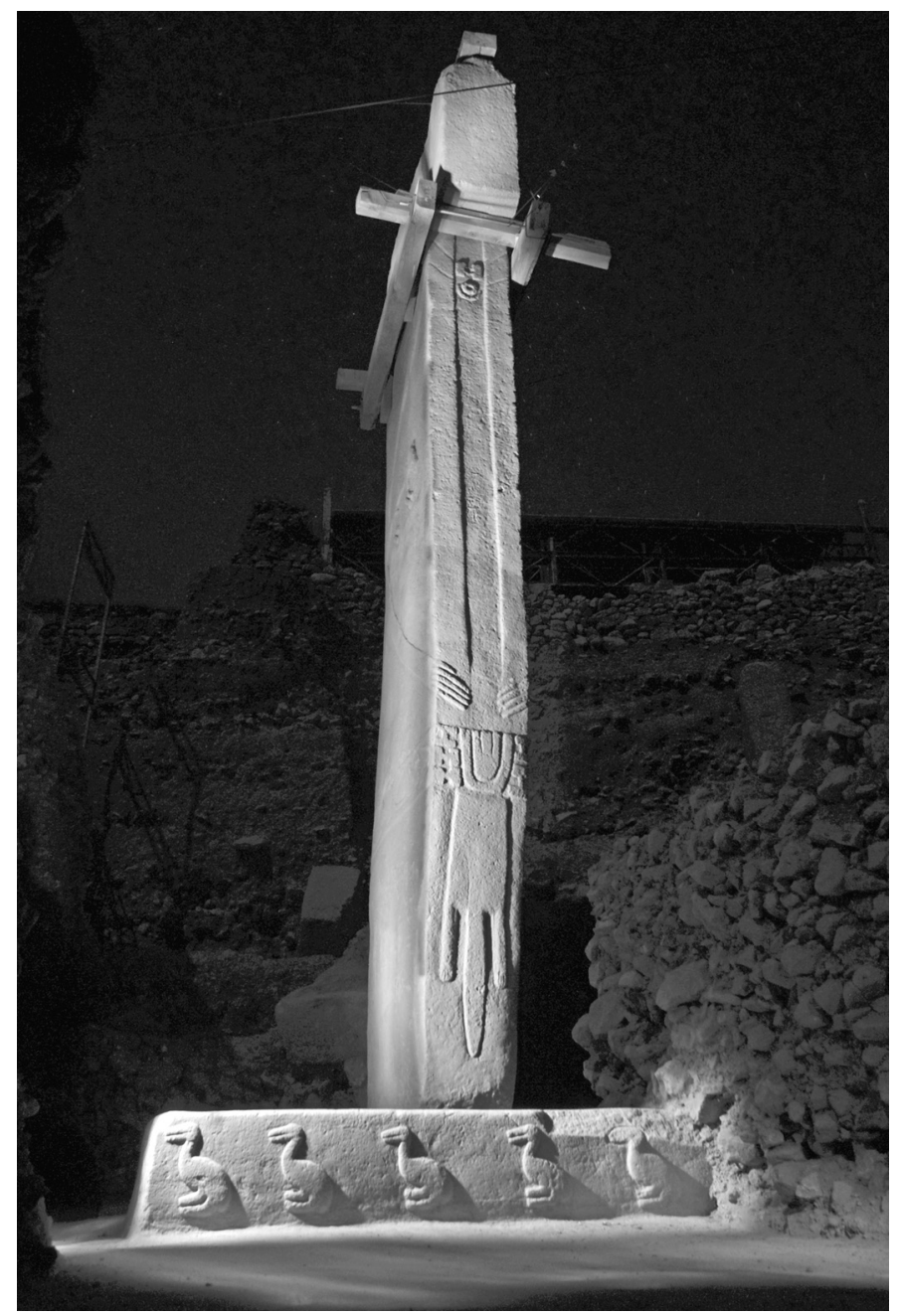

Gewissheit erlangen können. Bei der ältesten bekannten vollständig erhaltenen und lebensgroßen menschlichen Plastik, der Urfa-Statue, ist angesichts der hier nicht eindeutigen Erhaltung der in Flachrelief gezeichneten Details nicht völlig klar, ob die Hände das männliche Glied ergreifen, ob sie den Phallus präsentieren 7 . Doch bei der Kilisik-Statue mit zwei übereinander gereihten Individuen - ins Bild gesetzt sind jedenfalls deren Armpaare - präsentiert das obere der dargestellten Wesen offenbar den Phallus ${ }^{8}$. Auch beim kürzlich entdeckten sog. Totempfahl vom Göbekli Tepe begegnen wir dieser Arm- und 
Handhaltung9. Unter einem Raubtier befindet sich eine Person, deren Kopf von den Pranken des Raubtieres gehalten wird. Die Hände der Person sind auf den Bauch gelegt, ganz offenbar ohne etwas zu halten und ohne sich zu berühren. Auch die zweite Person, die unterhalb der ersten dargestellt ist, zeigt diese Arm- und Handhaltung. Zwischen den Händen befindet sich hier aber ein (vergleichsweise großes) Objekt. Es bleibt leider unklar, wie dieses zu deuten ist. Es könnte einen Kopf darstellen, so dass es sich um eine Geburtsszene handeln würde. Es könnte sich aber auch um die Darstellung des Skrotums handeln und eine Bruchstelle am oberen Rand des Objektes die Ansatzstelle des abgebrochenen Phallus anzeigen, den bei dieser Deutungsvariante die Hände ergreifen würden.

Das Phallus-Präsentieren kann wie das weibliche Äquivalent, das Schamweisen, als apotropäische Geste verstanden werden ${ }^{10}$. Die meisten der von Irenäus Eibl-Eibesfeld und Christa Sütterlin beschriebenen Darstellungen dieser Art wirken stark aggressiv auf den Betrachter. Erika Qasim verweist auf das weitverbreitete Vorhandensein dieses Gestus bei neolithischen Figurinen der Alten Welt ${ }^{11}$. Sie führt $u$. a. eine weibliche Darstellung aus der Hamangia-Kultur an, die die gleiche Arm- und Handhaltung wie die T-Pfeiler aufweist. Bemerkenswert, dass diese Figurine ohne Kopf und Gesicht, nur mit einem aus den Schultern erwachsenden langezogenen konischen Zapfen angefertigt wurde. Das Schamweisen ist bei diesen Figurinen mit auffallend großen Brüsten kombiniert, so dass Qasim hier in überzeugender Weise eine Verdopplung des Abwehrgestus, ein Verbindung von Brust- und Schamweisen sieht.

Ist es die apotropäische Haltung des Phallus- bzw. des Schamweisens, die trotz der Reduzierung des Gestus auf die Hände, trotz der Nichtdarstellung des Genitals den T-Pfeilern zukommt? Ist mit diesem Gestus die Bedeutung der T-Pfeiler gefunden und gehören die Kreisanlagen zu den Strategien zur Bewältigung von Angst, verkörpern die T-Pfeiler in Stein konservierte apotropäische Gesten?

Der Gedanke, dass auch die T-Pfeiler diesen Gestus einnehmen, ohne dass das Genital dargestellt wäre, kann nach der vollständigen Freilegung der beiden Zentralpfeiler der Anlage D ausgeschlossen werden. Hier fanden sich neben Armen, Händen und Stola noch weitere Details, die zunächst den Statuencharakter der Pfeiler unterstreichen und deutlich machen, wie sehr »Pfeiler« als Hilfsbegriff zu verstehen ist. Beide Pfeiler tragen einen Gürtel, an dem auf der Bauchseite ein Tierfell als Lendenschurz befestigt ist (Abb. 4). Auch wenn dieser die Genitalregion verdeckt und nicht unmittelbar erkennen lässt, welchem Geschlecht die Pfeilerwesen zugehören, so können wir nach unseren bisherigen ikonographischen Kenntnissen recht sicher sein, dass als Gürtelträger nur Männer in Frage kommen. Da die Hände sich aber über dem Gürtel und dem Lendenschurz befinden, ist mit Sicherheit auszuschließen, dass die Pfeilerwesen als phallische Bildnisse konzipiert waren.

9 Köksal-Schmidt-Schmidt 2010.

10 Eibl-Eibesfeld - Sütterlin 1992.

11 Qasim 2009. 
Somit lässt sich abschließend feststellen, dass die Frage nach dem Verhältnis von Bild zu umgebendem Raum klar beantwortet werden kann. Die T-Pfeiler und die darauf angebrachten Bilder definieren den Raum, sie selbst formieren sich zum architektonischen Gebilde. Welche Handlungen allerdings in diesem Raum vollführt wurden, welche Auswirkungen die Präsenz der Bilder auf die Art und Weise des Vollzugs der Handlungen hatte ist noch ungeklärt ebenso wie die Frage, inwieweit die Art und Weise der Gestaltung der Bilder und des Raumes eine Folge der dort vollzogenen Handlungen war. Der archäologische Interpret steht vor dem Problem, eine vor 12.000 Jahren kodierte Botschaft dekodieren zu wollen, eine Botschaft, die zudem nicht an ihn adressiert war. Bei der Deutungsarbeit stützen wir uns auf die Annahme, dass die Zeichen bewusst zur Kodierung einer Botschaft geschaffen wurden. Wir können deshalb zuversichtlich sein, dass bei der Fortführung der Ausgrabungen und der zu erwartenden zahlreichen Neufunde in dieser Frage bald bedeutende Schritte hin zu befriedigenden Antworten möglich sein werden und letztlich steht zu erwarten, dass auch die monumentalen Anlagen des Göbekli Tepe wie die vieler anderer Kulturen im Dienste der kulturellen Überwindung des Todes standen.

\section{Bibliographie}

\section{Bothmer-Plates et al. I989}

A. Graf von Bothmer-Plates - H.-M. Esen-Baur - D. Sauer, 1500 Jahre Kultur der Osterinsel. Schätze aus dem Land des Hotu Matua. Ausstellungskatalog Frankfurt a. M. (Mainz 1989).

\section{Bucak - Schmidt 2003}

E. Bucak - K. Schmidt, Dünyanın en eski heykeli, Atlas 127, ekim 2003, 36-40.

\section{Eibl-Eibesfeld - Sütterlin 1992}

I. Eibl-Eibesfeld - C. Sütterlin, Im Banne der Angst. Zur Natur- und Kunstgeschichte menschlicher Abwehrsymbolik (München 1992).

\section{Esen-Baur 1989}

H.-M. Esen-Baur, Megalithische Steinplastiken und monumentale Architektur, in: A. Graf von Bothmer-Plates - H.-M. Esen-Baur - D. Sauer, 1500 Jahre Kultur der Osterinsel. Schätze aus dem Land des Hotu Matua. Ausstellungskatalog Frankfurt a. M. (Mainz 1989) 87-108.

\section{Hauptmann 2000}

H. Hauptmann, Ein frühneolithisches Kultbild aus Kommagene, in: J. Wagner, (Hg.), Gottkönige am Euphrat. Neue Ausgrabungen und Forschungen in Kommagene, Sonderbände der Antiken Welt. Zaberns Bildbände zur Archäologie (Mainz 2000) 5-9.

\section{Heyerdal 1975}

T. Heyerdal, Die Kunst der Osterinsel. Geheimnisse und Rätsel (Gütersloh 1975). 


\section{Jursch 1952}

H. Jursch - I. Jursch, Hände als Symbol und Gestalt ${ }^{3}$ (Berlin 1952).

\section{Köksal-Schmidt - Schmidt 20 IO}

Ç. Köksal-Schmidt - K. Schmidt, The Göbekli Tepe »Totem Pole«. A First Discussion of an Autumn 2010 Discovery (PPN, Southeastern Turkey), Neo-Lithics 1, 2010, 74-76.

\section{Morenz - Schmidt 2009}

L. D. Morenz - K. Schmidt, Große Reliefpfeiler und kleine Zeichentäfelchen. Ein frühneolithisches Zeichensystem in Obermesopotamien, in: P. Andrássy - J. Budka F. Kammerzell (Hgg.), Non-Textual Marking Systems, Writing and Pseudo Script from Prehistory to Modern Times, Lingua Aegyptia - Studia monographica 8 (2009) 13-31.

Qasim 2009

E. Qasim, Frauenstatuetten: Zwei Gesten als Teil der Darstellung. Ein Beitrag zur Interpretation in: ArchaeNova e.V. (Hg.), Erste Tempel - Frühe Siedlungen. 12000 Jahre Kunst und Kultur. Ausgrabungen und Forschungen zwischen Donau und Euphrat, (Oldenburg 2009) 161-185.

\section{Schmidt 1995}

K. Schmidt, Investigations in the Upper Mesopotamian Early Neolithic: Göbekli Tepe and Gürcütepe, Neo-Lithics 2, 1995, 9-10.

\section{Schmidt 2009}

K. Schmidt, Göbekli Tepe - eine apokalyptische Bilderwelt aus der Steinzeit, Antike Welt 4, 2009, 45-52.

\section{Schmidt 20I0}

K. Schmidt, Göbekli Tepe - Sanctuaries of the Stone Age. New results of the Ongoing Excavations with a Special Focus on the Sculptures and High Reliefs, ${ }^{\text {th }}{ }^{\text {Neolithic }}$ Seminar Bodies, Rituals and Religions in Eurasian Early Prehistory, Documenta Praehistorica XXXVII, 2010, <http://arheologija.ff.uni-lj.si/documenta/pdf37/37_21.pdf>.

\section{Seitz 2006}

G. Seitz, Die Bildsprache des Buddhismus (Düsseldorf 2006).

\section{Wollenik 1982}

F. Wollenik, Abwehrhand und Drudenfuß - Felsbilder in Bayern. Unter Mitarbeit von Ebba Wendt, Maria Paulus, Peter Wörnle (Hallein 1982).

\section{Abbildungsnachweis}

Abb. 1. 4 : N. Becker, DAI; Abb. 2: I. Wagner, DAI; Abb. 3: K. Schmidt, DAI. 